\title{
Case-based lung image categorization and retrieval for interstitial lung diseases: clinical workflows
}

\author{
Adrien Depeursinge - Alejandro Vargas • Frédéric Gaillard • \\ Alexandra Platon - Antoine Geissbuhler • Pierre-Alexandre Poletti • \\ Henning Müller
}

Received: 17 January 2011 / Accepted: 9 May 2011 / Published online: 1 June 2011

(C) CARS 2011

\begin{abstract}
Purpose Clinical workflows and user interfaces of imagebased computer-aided diagnosis (CAD) for interstitial lung diseases in high-resolution computed tomography are introduced and discussed.

Methods Three use cases are implemented to assist students, radiologists, and physicians in the diagnosis workup of interstitial lung diseases.

Results In a first step, the proposed system shows a threedimensional map of categorized lung tissue patterns with quantification of the diseases based on texture analysis of the lung parenchyma. Then, based on the proportions of abnormal and normal lung tissue as well as clinical data of the patients, retrieval of similar cases is enabled using a multimodal distance aggregating content-based image retrieval (CBIR) and text-based information search. The global system leads to a hybrid detection-CBIR-based CAD, where detection-based and CBIR-based CAD show to be complementary both on the user's side and on the algorithmic side. Conclusions The proposed approach is in accordance with the classical workflow of clinicians searching for similar cases in textbooks and personal collections. The developed system enables objective and customizable inter-case similarity assessment, and the performance measures obtained
\end{abstract}

A. Depeursinge $(\varangle) \cdot$ F. Gaillard · H. Müller

MedGIFT Group, Business Information Systems,

University of Applied Sciences Western Switzerland (HES-SO),

Techno Ark 3, 3960 Sierre, Switzerland

e-mail: adrien.depeursinge@hevs.ch

A. Vargas · A. Geissbuhler

Service of Medical Informatics,

University and University Hospitals of Geneva (HUG),

Rue Gabrielle-Perret-Gentil 4, 1211 Geneva 14, Switzerland

A. Platon · P.-A. Poletti

Service of Emergency Radiology, HUG, Geneva, Switzerland with a leave-one-patient-out cross-validation (LOPO CV) are representative of a clinical usage of the system.

Keywords Computer-aided diagnosis (CAD) . Content-based image retrieval (CBIR) .

Interstitial lung diseases - Texture analysis .

Clinical worklows

\section{Introduction}

The number of images produced per day in most modern hospitals followed an exponential growth during the last decade. The mature field of imaging physics brought a large variety of essential diagnosis tools to the clinicians [33]. As a consequence, the explosion of the quantity and variety of medical visual information has the undesirable effect to overwhelm the radiologists with image interpretation tasks. About 15 years ago, the digital form of medical images along with their standardized storage gave birth to a new domain at the crossing of computer vision and medicine: image-based computeraided diagnosis (CAD). As a response to the imaging physics breakaway, image-based CAD systems have the potential to improve both the effectiveness and efficiency of the radiologists $[9,10]$. Goal of CAD is to use computer vision to assist radiologists in focusing their attention on diagnostically interesting events [11]. The CAD system can be used as first reader in order to improve the radiologists' productivity and reduce reading fatigue $[24,26]$. Whereas the radiologists' ability to interpret visual information is likely to change based on the domain-specific experience, human factors and time of the day, computerized classification of lung tissue patterns is $100 \%$ reproducible and can be quantitative and not only qualitative. 


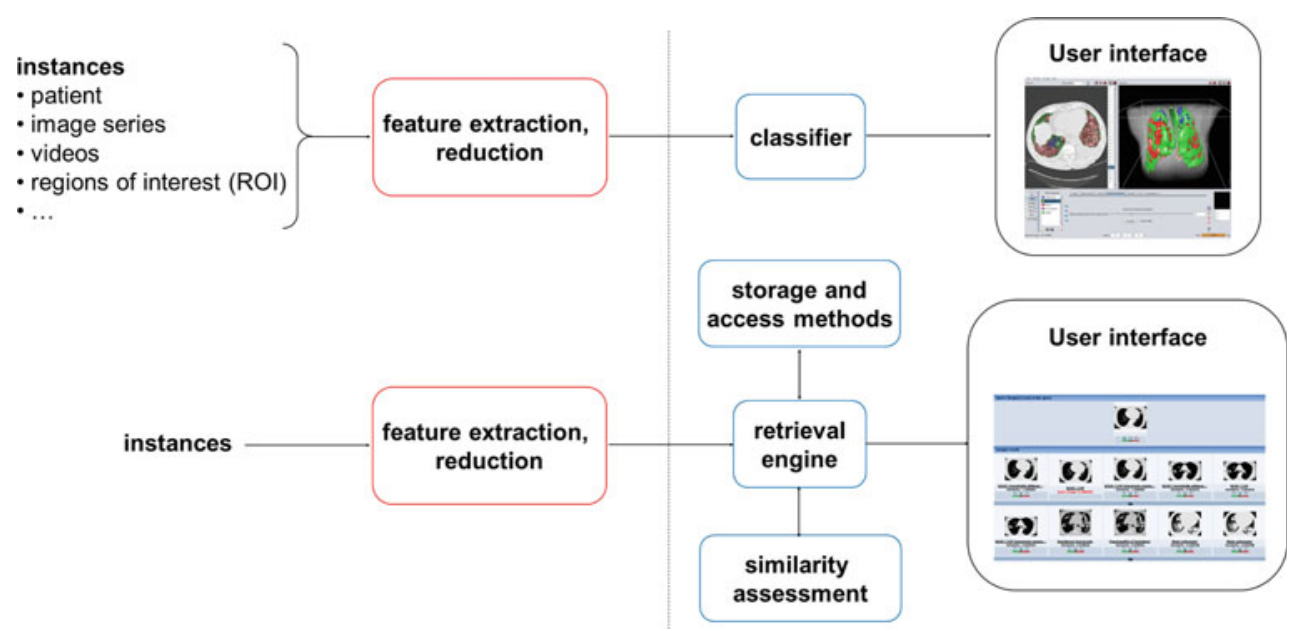

Fig. 1 Flowchart showing the similarities and differences between detection-based CAD (top) and CBIR-based CAD (bottom)

CAD was also proposed in several fields of medicine with non-visual systems such as $\mathrm{QMR}^{1}$ (quick medical reference) or $\operatorname{ILIAD}^{2}[12,19,23,39]$. Early studies on computerized analysis of medical images (mostly in X-ray imaging) were reported in the 1960s $[18,22]$. These systems aimed at replacing the radiologists as the originators relied on the assumption that computers are better at performing certain tasks than are human beings. However, it quickly became clear that physicians and radiologists have to take the final decision, and outputs of CAD systems must be used as "second opinions" and information providers [11,21].

Recently, CAD systems have been used in clinical routine in the rather mature field of cancer screening in mammograms and allowed to improve the detection of non-palpable cancerous masses $[15,38]$. Image-based CAD systems are expected to be introduced into clinical routine for several other organs such as the chest, colon, brain, liver, skeletal and vascular systems $[1,9,26]$.

Mainly two approaches are proposed in the literature for assessing image-based computerized diagnostic aid: detection-based CAD and content-based image retrieval (CBIR)based CAD. The former exhaustively analyzes image series to detect and characterize (or localize) anomalies whereas the latter aims at retrieving images based on the visual similarity. Detection-based CAD (also called CADe for computer-aided detection in the literature) can quickly provide an overview of the diseased regions, which reduces the risk of omission and ensures the reproducibility of the diagnosis by drawing the radiologists' attention to diagnostically interesting events in medical image series. The classical scheme for event detection and quantification in medical image series is depicted in Fig. 1 (top). The instances

\footnotetext{
$\overline{1}$ http://www.openclinical.org/aisp_qmr.html, as of April 8, 2011.

2 http://www.openclinical.org/aisp_iliad.html, as of April 8, 2011.
}

constitute the input of the CAD system and can be patients, image series, videos, and regions of interest (ROI). From the instances, a set of features aiming to compactly describe the diagnostically relevant visual content is extracted. When required, a feature reduction is applied to cut down the number of features. The resulting set of attributes constitutes the feature space in which the classifier draws decision boundaries to predict the class(es) of the instance(s) that are displayed in the appropriate format to the user via a graphical user interface (GUI). CBIR-based CAD delivers quick and precious information for diagnosis aid and treatment planning through example cases with confirmed diagnosis. The automatic indexation of medical visual content with CBIR enables systematic storage of the ever-increasing production of medical images in today's modern hospitals. Medical data repositories represent potentially rich knowledge bases. However, the access to medical image series in the picture archiving and communication system (PACS) is most often based on the patient identification number, which is not suitable to search for similar cases based on a disease or on the visual similarity of the images. Thereby, CBIR allows to quickly find similar images according to objective criteria in large image collections to assist the radiologists in their diagnosis workup $[25,30]$. Concepts for integration of CBIR into medical practice were often proposed in the literature $[24,27,41]$.

However, few CBIR systems have been integrated and evaluated in clinical practice. A major challenge is to find features and distance measures that are matching the clinicians' requirements according to a particular application. The discrepancy between the user's intentions when looking for a particular image and the visual information that the features are able to model is called the "semantic gap" in the literature [35]. It is usually a bottleneck in medical CBIR. Nevertheless, the few CBIR-based CADs evaluated 
in clinical practice showed very promising results as that they can be accepted by the clinicians as a helpful and easy-to-use tool and allow significant improvement in the diagnosis accuracy, especially for little experienced radiologists $[2,3,16,32]$. A possible extension to CBIR is to carry out case-based retrieval. Most often, the clinician actually looks for similar cases as he considers the image within the context of a patient with a personal history, findings on the physical examination, laboratory tests, etc. At last, CBIR can be complementary to event detection where the features can be used both for the categorization of the image series and for the retrieval of similar instances. Conceptually, both CAD configurations rely on visual features describing the visual content of the images. Based on a visual feature space, the detection-based CAD decides to which predefined class (e.g. "normal" or "diseased") the input instance (i.e., image or image region) belongs, whereas CBIR-based CADs output a similarity score between two instances from which a ranked list of images can be built (usually showing the few most similar instances). The two approaches are depicted in Fig. 1.

Use cases, visualization and software

Until recently, film-based radiological images allowed visualizing organs only via $2 \mathrm{D}$ projections of three-dimensional organs. The digital capture of medical visual information enables advanced visualization techniques. Threedimensional colored objects with motion correspond to our visual perception of the world. Several DICOM (Digital Imaging and Communications in Medicine) viewers integrated tools for representing four-dimensional visual color information [29]. A notable example is the open-source software OsiriX ${ }^{3}$ [28] based on the visualization toolkit ${ }^{4}$ (VTK) [31]. Using virtual reality was proposed by [13]. The visualization tools aim at representing the most information at once in order to reduce the reading time and to assess global views of organs synthesizing visual information to ease the diagnosis workup.

\section{Computer-aided diagnosis for interstitial lung diseases}

Interstitial lung diseases (ILD) constitute a heterogeneous group with more than 150 disorders of the lung tissue from various causes leading to breathing dysfunction. Many of them are rare and present unspecific symptoms such as altered pulmonary functions and cough. Diagnosing ILDs is a difficult task for non-specialists and is based on observations of clinical, physiologic, pathologic manifestations as well as radiologic findings [17]. The high-resolution computed tomography (HRCT) imaging protocol is commonly used as

\footnotetext{
3 http://www.osirix-viewer.com/, as of April 8, 2011.

${ }^{4}$ http://www.vtk.org/, as of April 8, 2011.
}

it enables accurate visual assessment of the lung parenchyma by providing three-dimensional images at a sub-millimetric resolution in axial slices. The interpretation of HRCT images of the chest showing disorders of the lung tissue associated with ILDs is time-consuming and requires experience as the various diagnoses of ILDs can be differentiated only by subtle changes in the lung parenchyma, with characteristic localization within the lung anatomy [40].

Several CAD approaches are proposed in the literature to assist the radiologists in the HRCT interpretation tasks. Most of the studies are investigating automated detection and quantification of the lung parenchyma $[14,34,37]$, whereas few studies used CBIR as diagnosis aid $[20,32,36]$. To our knowledge, none are able to retrieve similar ILD cases based on the histological diagnosis $[6,8]$.

In this work, clinical workflows and user interfaces of a hybrid detection-CBIR-based CAD system based on texture analysis of high-resolution computed tomography (HRCT) images of patients affected with interstitial lung diseases (ILDs) are proposed and discussed. Based on the output of a detection-based CAD, a multimodal similarity measure is used to enable case-based retrieval, yielding a hybrid detection-CBIR-based CAD system. Using cases for database browsing, three-dimensional automated categorization of the lung tissue in HRCT and case-based retrieval are defined and implemented in a rich Internet application (RIA) with graphical user interfaces (GUIs).

The paper is structured as follows: in section "Material and methods", the dataset, the three use cases and the software used are described. The clinical workflows and associated GUIs of the proposed hybrid detection-CBIR-based CAD are detailed and depicted in section"Results" and discussed in section "Discussions". Conclusions are given in section "Conclusions".

\section{Materials and methods}

The dataset used for the construction and evaluation of the CAD system is described in the first part of this section. Then, three use cases and their implementation are detailed.

\section{Collection of ILD cases}

A multimedia library of ILD cases was built at the University Hospitals of Geneva (HUG) [7]. It contains a selection of clinical parameters associated with ILDs and HRCT image series with annotated regions and aims at providing ground truth for the evaluation of the CAD algorithms and constitutes a reference library for teaching. The retrospective collection of the cases has been approved by the appropriate ethics committee. A total of 128 histologically proven cases of 13 frequent ILDs as well as healthy cases are currently captured in the 
database. Among them, 108 have an annotated HRCT image series corresponding to the correct period of care. A total of 41.65 liters of lung tissue were annotated by two radiologists with 15 and 20 years of experience.

\section{Use cases and software}

The definition of use cases adapted to the needs of the radiologists in a particular context is an important part for the success of the CAD. Three use cases are defined to optimize diagnosis aid for ILDs:

1. navigation through the multimedia database records for teaching and learning purposes,

2. automated $3 \mathrm{D}$ categorization of the lung tissue in HRCT image series,

3. case-based retrieval.

The second and the third use cases are strongly interdependent, yielding a hybrid detection-CBIR-based CAD system (see section "3D lung tissue categorization and case-based retrieval"). The three use cases were implemented in a RIAbased on Adobe FLEX ${ }^{5}$ and Java Applet ${ }^{6}$ using Java3D ${ }^{7}$. The Java Applet is based on a modified version of $\mathrm{YaDiV}^{8}$, an open-source DICOM viewer enabling advanced 3D visualizations and rendering. The goal was to develop a fully web-based application, so it can be used by either students or clinicians and from various places inside the hospitals. All software to be installed on clinical desktops at the University Hospitals of Geneva has to go through a tedious administrative task. As soon as a Java virtual machine and Java3D are installed, the RIA can be accessed directly by typing the corresponding uniform resource locator (URL) in a web browser. Once logged in, the user can choose between browsing the multimedia database using exact text search and analyzing a new case along with retrieving similar patients (see Fig. 2).

\section{Results}

This section details the clinical workflows used either for browsing the database or for analyzing a new case and retrieving similar ones. The use case for browsing the database is detailed in section "Multimedia database browsing". The workflow for the analysis of lung tissue of a new case combined with the case-based retrieval is described in section "3D lung tissue categorization and case-based retrieval".

\footnotetext{
5 http://www.adobe.com/fr/products/flex/, as of April 8, 2011.

6 http://java.sun.com/applets/, as of April 8, 2011.

7 http://www.java3d.org/, as of April 8, 2011.

8 YaDiV: Yet Another DIcom Viewer, http://www.welfenlab.de/en/ research/projects/yadiv/, as of April 8, 2011.
}

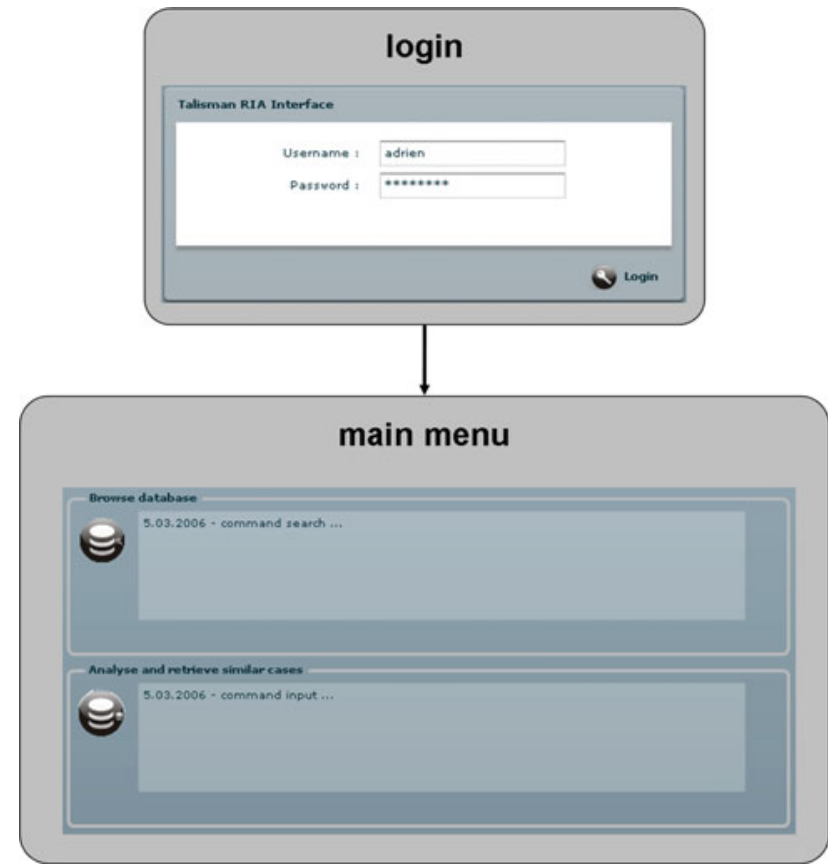

Fig. 2 Welcome menus of the RIA. Once logged in, the user can choose between navigating through database records (see Fig. 3) and analyzing a new case along with retrieving similar patients (Fig. 9)

\section{Multimedia database browsing}

The workflow for the navigation through the database records is depicted in Fig. 3. This use case is mostly intended for teaching where students, interns, and little experienced radiologists can consult the typical clinical and radiological manifestations of ILD diagnoses.

The user can perform a text-based search by defining the target value of each clinical parameter as shown in Fig. 4. And and $\mathrm{Or}$ combination rules are available to favor either precision or recall of the retrieved information. Based on the query, an exact text search is performed, and the returned cases must have exact corresponding values of the clinical parameters. For instance, if the fields "Diagnosis", "Age" and "Gender" are filled with values "tuberculosis", "58" and "f" respectively, and And rules are used, only 58 years-old female patients with tuberculosis will be retrieved. For all parameters with continuous values, inequality rules are also available. For instance, all cases with age greater or equal to 58 years old can be retrieved. The retrieved patients are listed in a dynamic page that allows to preview the clinical parameters organized in three levels of importance (see Fig. 5). The important levels of each clinical parameter vary based on the diagnosis of the case in order to show the most relevant parameters in the second level. A pop-up window showing a preview of the HRCT image series can be obtained by clicking on the thumbnail image displayed on the right side of each case. As soon as the user wants to access the full case data 
Fig. 3 Workflow of the RIA for the navigation in the database using exact text search
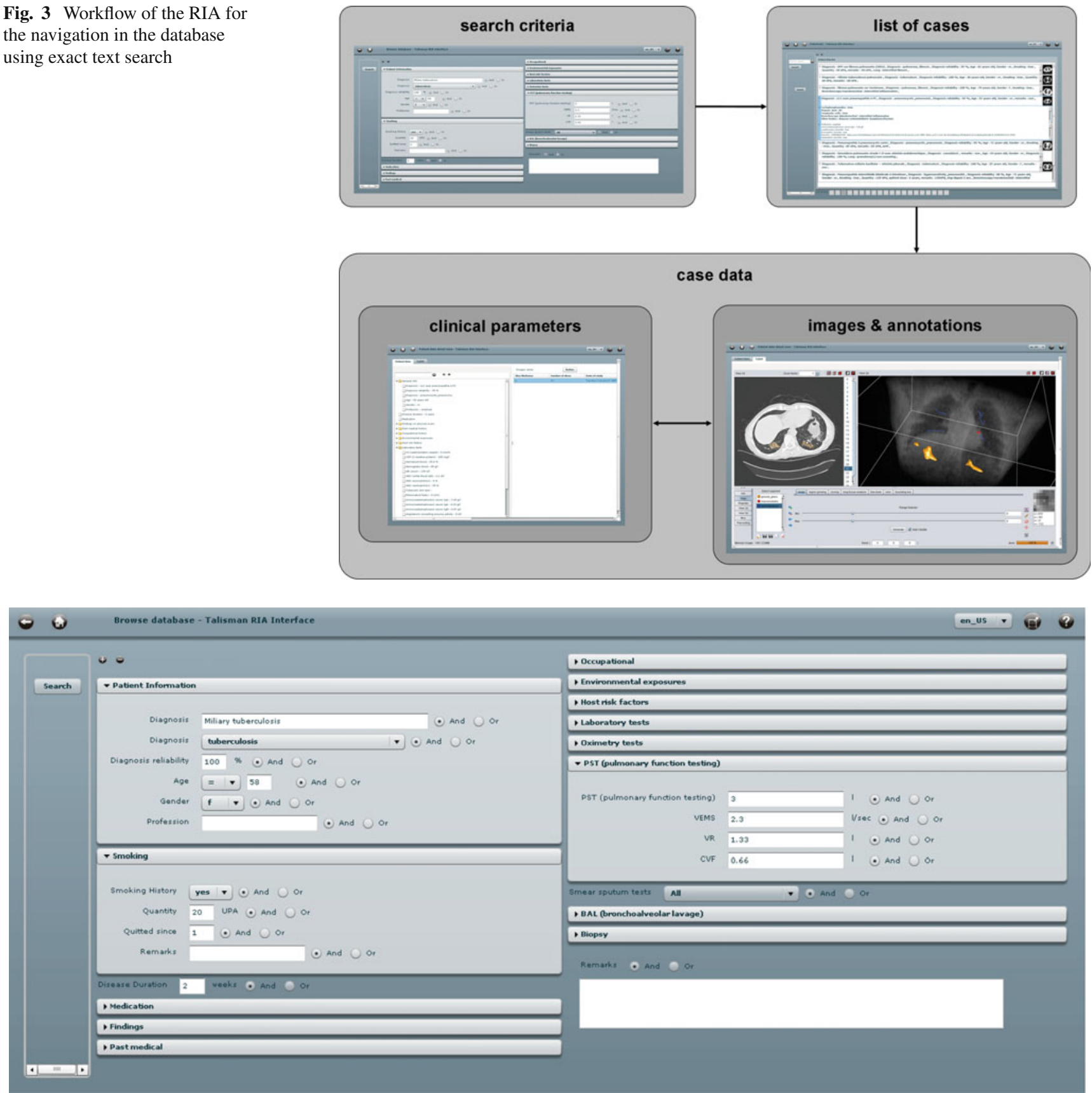

Fig. 4 Form containing the clinical parameters for querying ILD cases using exact text search. And and Or combination rules are available

based on the previewed information, a "Detail" button can be clicked to open a new page that displays every available clinical parameter using a tree-like structure (see Fig. 6). On the right panel of this page, the HRCT image series are listed by the date of the study. By double-clicking on an image series, the YaDiV Java Applet is launched and opens the requested image series along with the associated annotated regions (see Fig. 7). 2D and 3D views are available to navigate through the image series and visualize the delineated pathological lung tissues. The user can switch between clinical parameters and
HRCT images by clicking either on the "Patient data" tab or the "YaDiV" tab. In order to perform a new search or to return to the main menu, the button showing a backward arrow on the top left of each page can be used.

\section{D lung tissue categorization and case-based retrieval}

The second use case implements the analysis of the image series of a new case and case-based retrieval. The main components of the hybrid detection-CBIR approach are detailed 


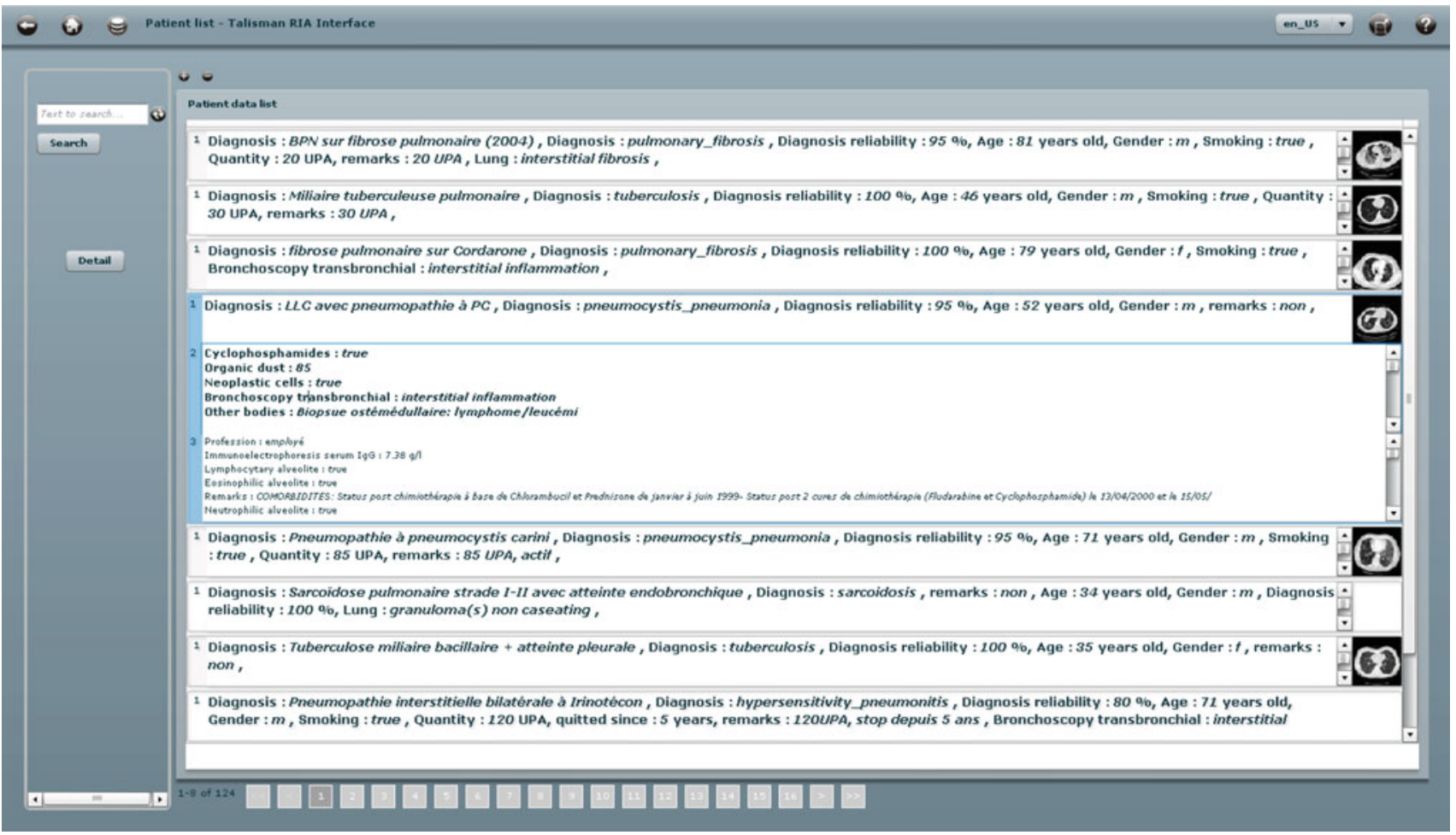

Fig. 5 List of cases returned by an exact text search. The user can preview the clinical parameters of the patients with three levels of importance. Levels of importance are varying based on the diagnosis

in Fig. 8. In a first step, the clinician can run the threedimensional categorization of the lung tissue on the undiagnosed incoming HRCT image series in order to obtain a 3D map of the lung tissue. In a second step, the clinician can retrieve similar cases from the multimedia database based both on the respective volumes of the previously segmented lung tissue sorts and on the value of the clinical parameters [5]. The workflow for the categorization of the lung tissue and case-based retrieval is detailed in Fig. 9. Starting from the page for submitting the query (top left image in Figs. 9 and 12), the user can either categorize lung tissue and fill clinical parameters to retrieve similar cases based on both visual features and text or skip the image analysis step to retrieve cases based only on the clinical parameters. By clicking on the button showing a 3D map of lung tissue (see Fig. 12), the YaDiV Java Applet is launched and the user can perform the 3D categorization of the lung tissue by opening an HRCT image series, segmenting the lung volumes and running the blockwise classification. The button showing a pie chart (see Fig. 12) is used for the retrieval of similar cases.

\section{$3 D$ categorization of lung tissue}

The use case implementing the categorization of the lung tissue is composed of the segmentation of the lung volumes in a first step and of the blockwise classification of the lung tissue in a second step.

Segmentation of lung volumes The segmentation of the lung volumes is a prerequisite for the categorization of the lung tissue. It consists of a 3D region growing followed by a closing operation. The $3 \mathrm{D}$ region growing routines are already available in the original distribution of YaDiV. A tab was added for the closing operation (see Fig. 10). When required, the user can manually edit the lung mask. The lung mask can be visualized in 2D and 3D to verify the completeness of the segmented volume.

Blockwise classification of the lung tissue As soon as the user is satisfied with the segmentation of the lung volumes, the routines for the categorization of the lung tissue can be run from the "lung tissue analysis" tab that was added to YaDiV. The distance separating the centers of $32 \times 32$ blocks can be specified. The "Generate" button runs the feature extraction and classification of each block belonging to the lung mask. Features used rely on the characterization of the grey-levels in Hounsfield Units (HU) as well as texture properties described using a wavelet frame transform specifically designed for lung tissue analysis [8]. Thanks to the wavelet frame transform avoiding downsampling of the images, the values of the wavelet coefficients can be accessed through every scale 


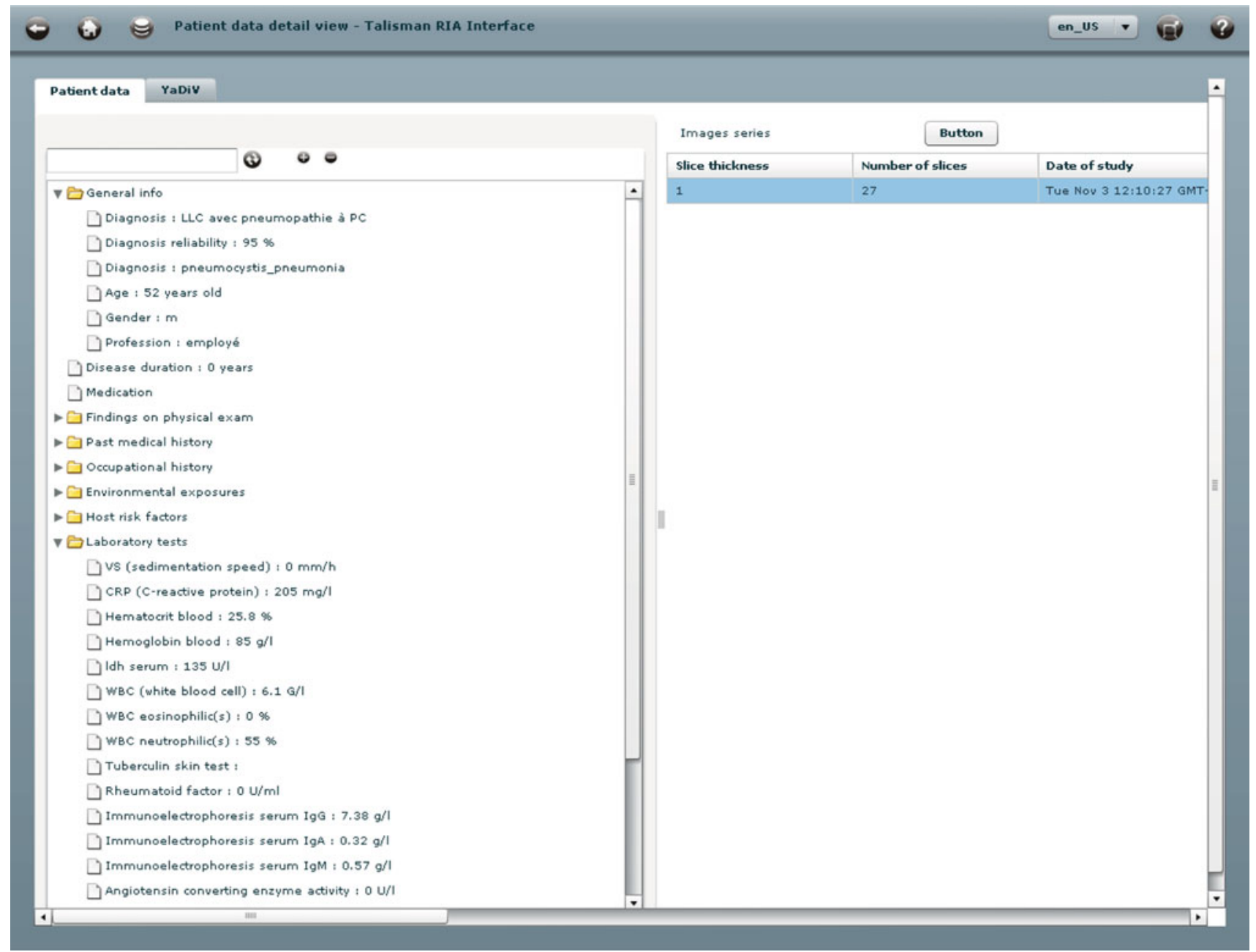

Fig. 6 Detailed view of a selected case: clinical parameters. This page is reached from the button "Detail" of Fig. 5

using identical $(x, y, z)$ coordinates as it is the case for the grey-level values. For each block, the corresponding feature vector contains bins of a grey-level histogram in $\mathrm{HU}$ along with parameters of Gaussian mixture models for each iteration of the wavelet transform and is classified using previously trained support vector machines (SVM). The result of the categorization is displayed using a three-dimensional map of the lung tissue that can be visualized using the 2D and $3 \mathrm{D}$ views of $\mathrm{YaDiV}$ as depicted in Fig. 11. The volumes in liters of each tissue type can be obtained by clicking on the corresponding segment.

\section{Similar case retrieval}

The user can submit the query for the retrieval of similar cases by clicking on the button showing a pie chart (see Fig. 12). If an image series was previously categorized, the query will be based both on visual and clinical features. Alternately, the retrieval will be based on the clinical parameters only.
The multimodal inter-case distance $d_{M}$ is based on a linear combination of a content-based distance $d_{\text {visual }}$ and two distances based on clinical parameters $d_{\text {param } 1,2}$ [6]:

$d_{M}=a_{1} d_{\text {visual }}+a_{2} d_{\text {param } 1}+a_{3} d_{\text {param } 2}$,

with $a_{j}$ being the weights of each modality. $d_{v i s u a l}$ is defined as the $l_{2}$-Euclidean distance in the space of the differences $\delta$ of percentages of the tissue types:

$d_{v i s u a l}=\sqrt{\delta_{h}^{2}+\delta_{e}^{2}+\delta_{g}^{2}+\delta_{f}^{2}+\delta_{m}^{2}}$,

where $h$ stands for healthy, $e$ for emphysema, $g$ for ground glass, $f$ for fibrosis and $m$ for micronodules and $d_{\text {param } 1,2}$ are the $l_{2}$-Euclidean distance in terms of clinical parameters of importance 1 and 2, respectively. $d_{\text {visual }}$ and $d_{\text {param } 1,2}$ are normalized before being combined in Eq. (1). Normalization is achieved by mapping minimum and maximum values to 0 and 1, respectively. Two pairs of lungs having identical proportions of lung tissue types will have a distance of 0 . A maximal distance of 1 is attributed if two pairs of lungs have 


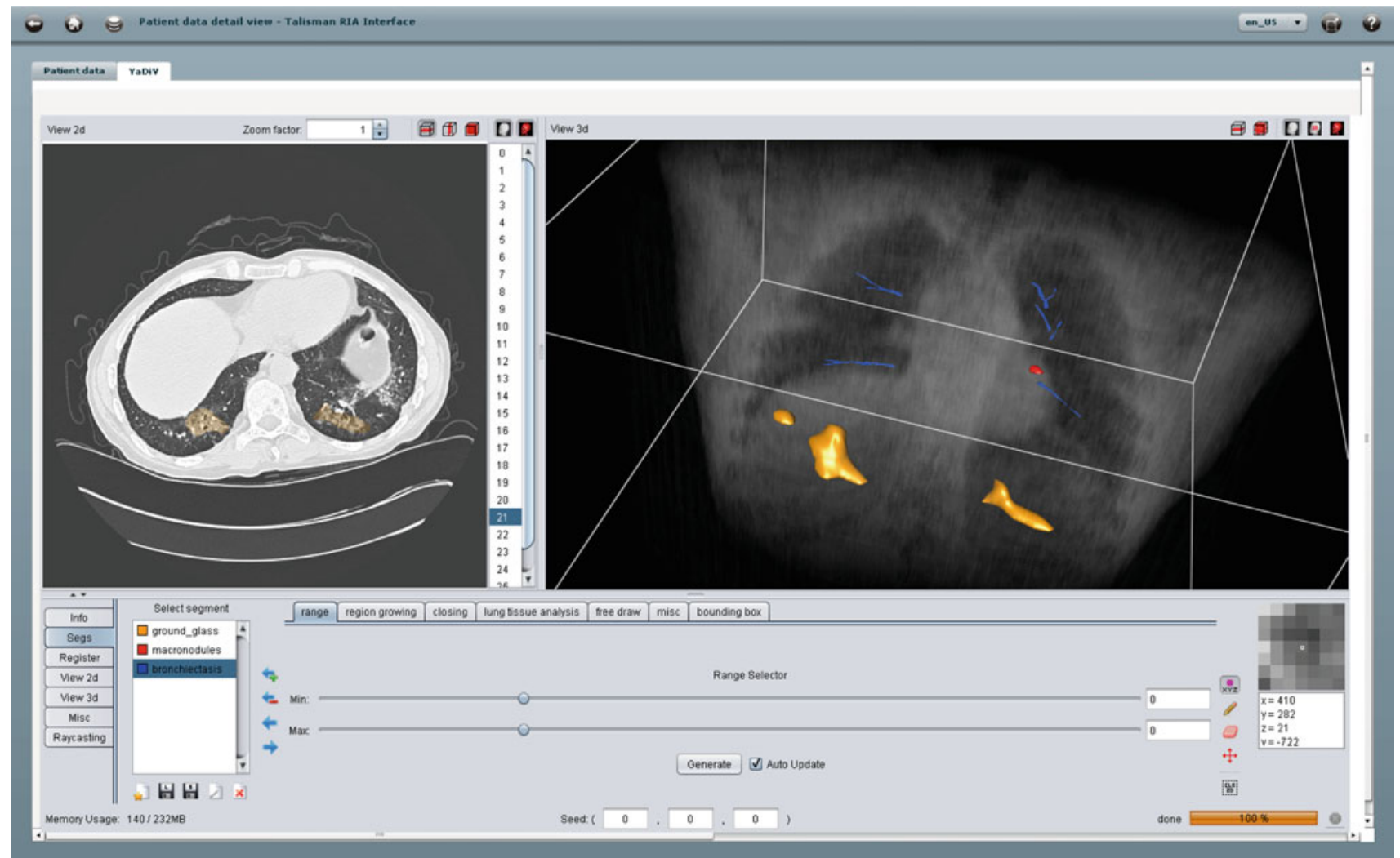

Fig. 7 Detailed view of a selected case: image series and annotations. 2D and 3D views are available to visualize the ROIs. YaDiV was adapted and embedded in a Java Applet

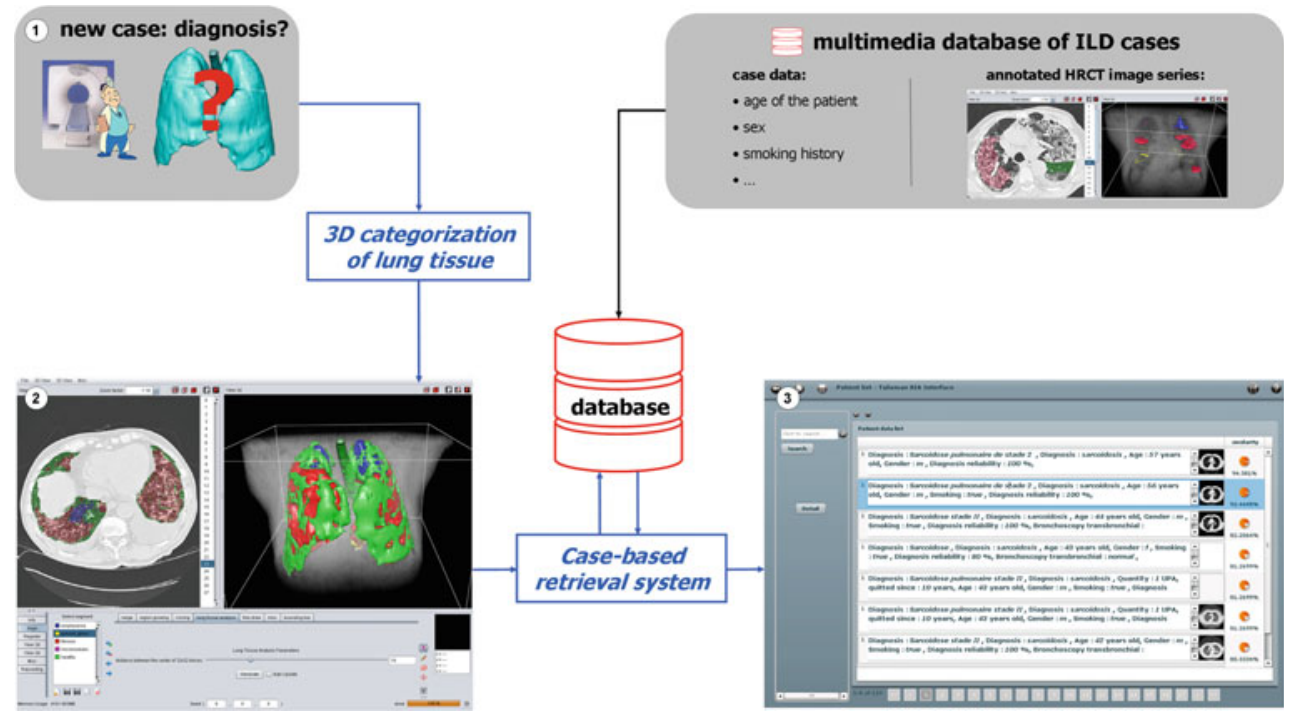

Fig. 8 Main components of the hybrid (detection-CBIR-based) CAD system

$100 \%$ of their lung tissue in two different categories. Linear combinations of distances showed to allow for efficient combination of text and visual modalities in [4]. A grid search for optimal weights of the modalities in Eq. (1) is carried out with $a_{j} \in[0: 50[$.
The ranked list of retrieved cases shown in Fig. 13 contains the same functionalities as the list of cases used in the use case for browsing the database in Fig. 5. A pie chart was added to display the similarity measure in percent for each case. Detailed views of the selected case identical to 


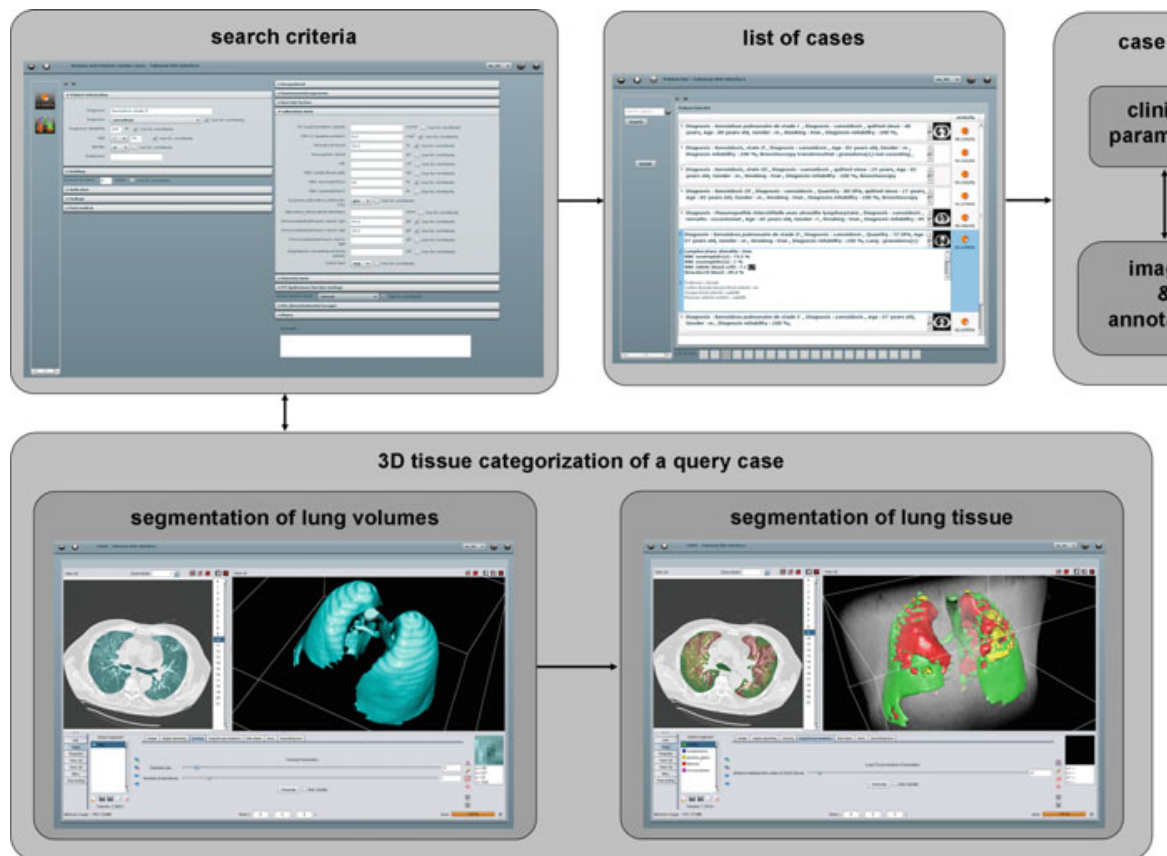

Fig. 9 Workflow of the RIA for the lung tissue categorization of a new case and the retrieval of similar patients. The query can be based both on specified clinical parameters and on the volumes of the segmented tissue types

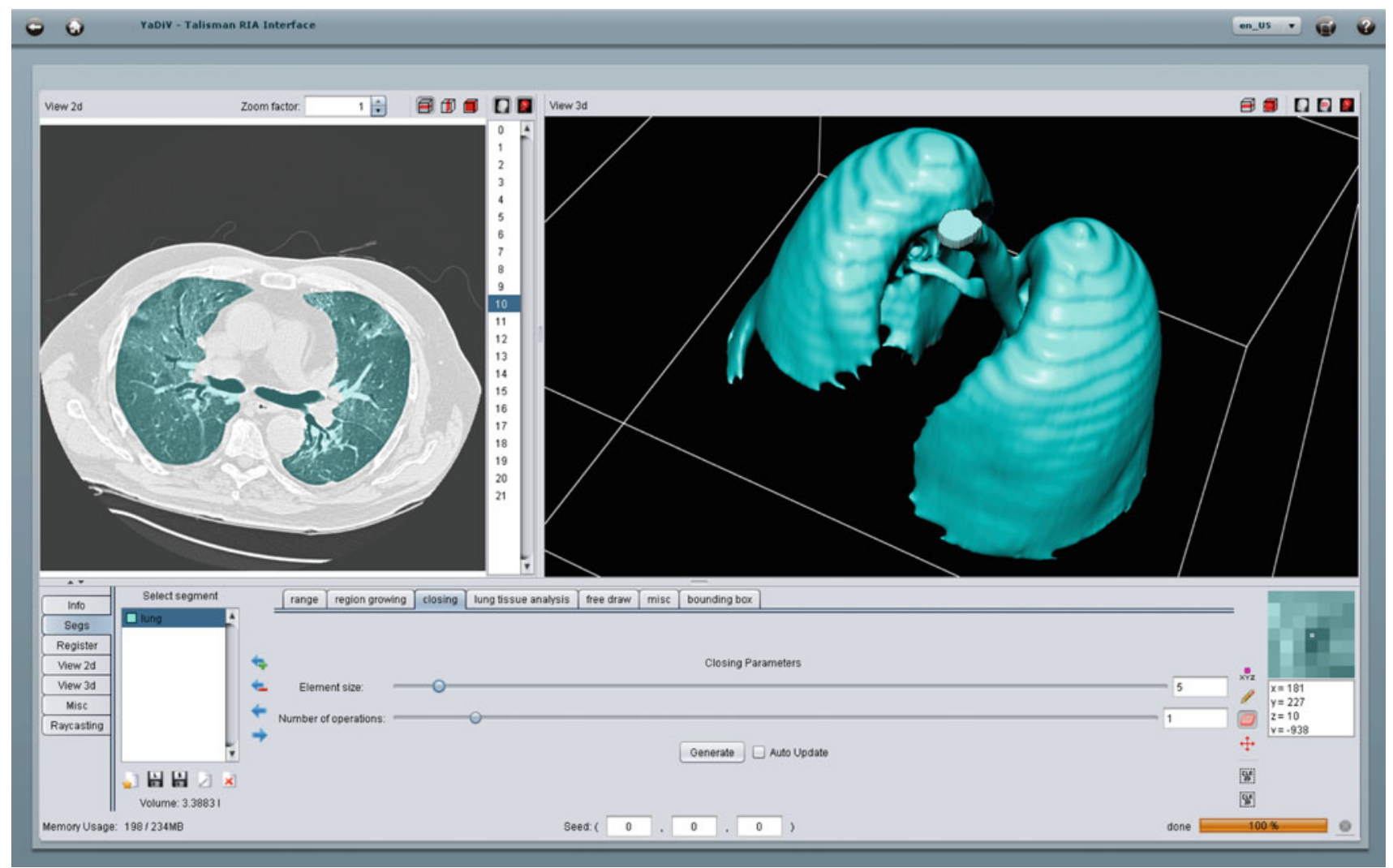

Fig. 10 Segmentation of the lung volumes using YaDiV. A "closing" tab was added to YaDiV to perform a closing operation after the region growing 


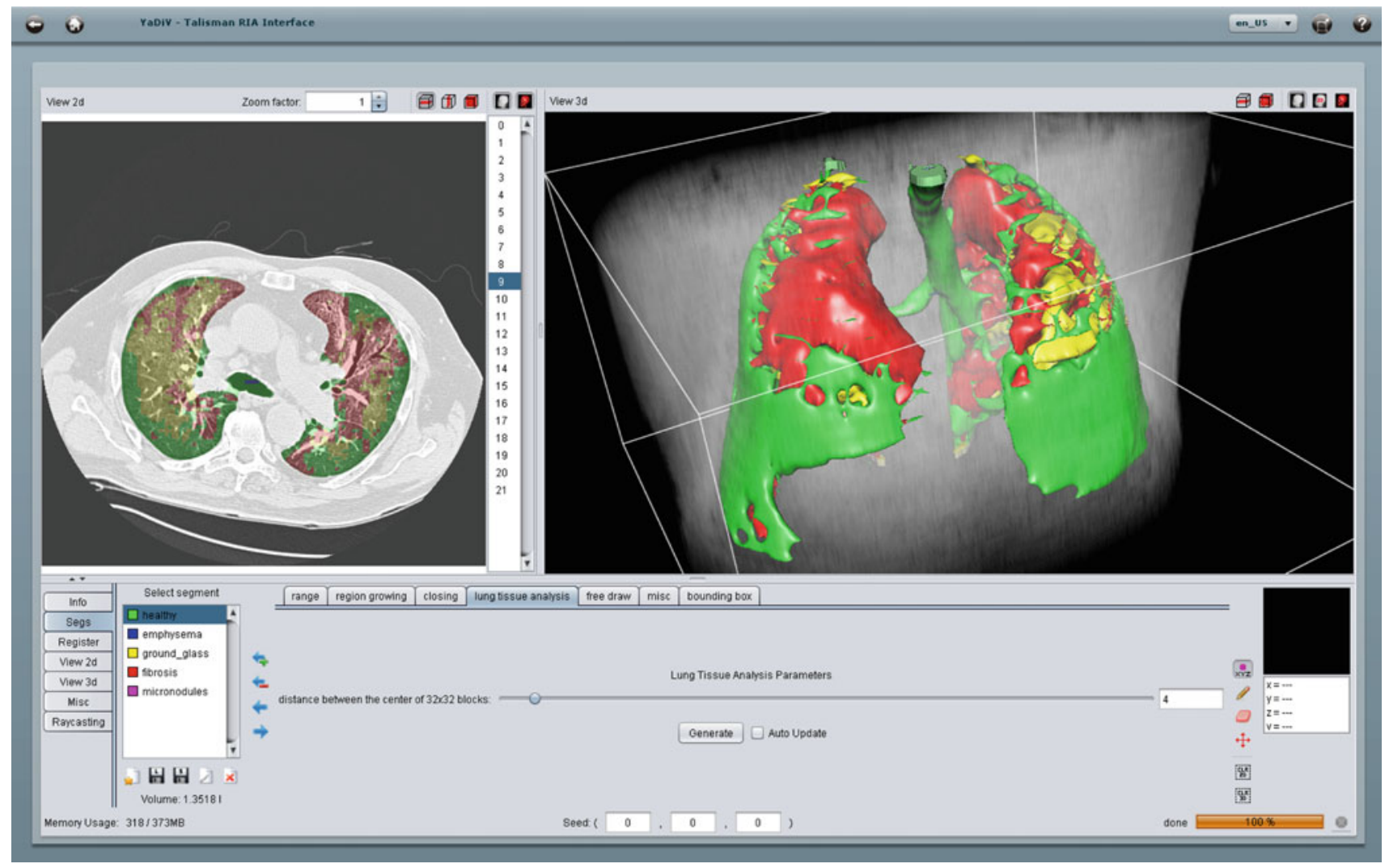

Fig. 11 Segmentation of the lung tissue using YaDiV. A "lung tissue analysis" tab was created to run the blockwise feature extraction and classification. The distance between the centers of the blocks can be tuned

the one used in the "browse" use case (see Figs. 6 and 7) can by obtained by clicking on the "Detail" button. Retrieval examples using $d_{\text {visual }}$ only are shown in Fig. 14 .

\section{Discussions}

The first part of this section discusses the proposed clinical workflows constituting the three use cases. A general discussion on the proposed hybrid detection-CBIR-based CAD system can be found at the end of the section.

The use case for multimedia database browsing enables easy access to the wealth of the collection of ILD cases. Efforts were taken to not overwhelm the user with information, where clinical parameters are displayed according to three degrees of importance varying based on the diagnosis and using tree-like structures. 3D access to full imaging data and annotated regions is enabled using the embedded $\mathrm{YaDiV}$ applet allowing traditional axial, coronal and sagittal views as well as volumetric navigation and rendering. With this use case, students, interns, and little experienced radiologists can consult the typical clinical and radiological manifestations of ILD diagnoses but also realize their variabilities through real cases. This use case is recommended to be used along with textbooks and literature such as $[17,40]$ that describe the fundamental mechanisms of the studied histological diagnosis. The success of this use case strongly depends on the database used, in which the number of cases per histological diagnoses varies from 1 to 40 , with a mean of 9.85 .

The second and third use cases are designed for radiologists and other physicians. The 3D categorization of the lung tissue provides a first lecture and second opinion of the HRCT scan and is thus dedicated to assist radiologists. On the one hand, this map highlights diagnostically useful events in the image series and thus reduces the risk of omission of important lesions of the lung tissue. On the other hand, it provide first insights into the potential histological diagnosis of the patient under investigation by providing the respective volumes of five lung tissue types ${ }^{9}$ that are associated with most of the ILDs. A leave-one-patient-out crossvalidation (LOPO CV) based on blocks as instances was used to assess the performance of the blockwise classification, which showed an arithmetic mean of $73.6 \%$ with high class specificity as the geometric mean of class-specific accuracies reaches $89.4 \%$ [8]. Classification performance is detailed in Table 1.

\footnotetext{
${ }^{9}$ Healthy, emphysema, ground glass, fibrosis and micronodules.
} 


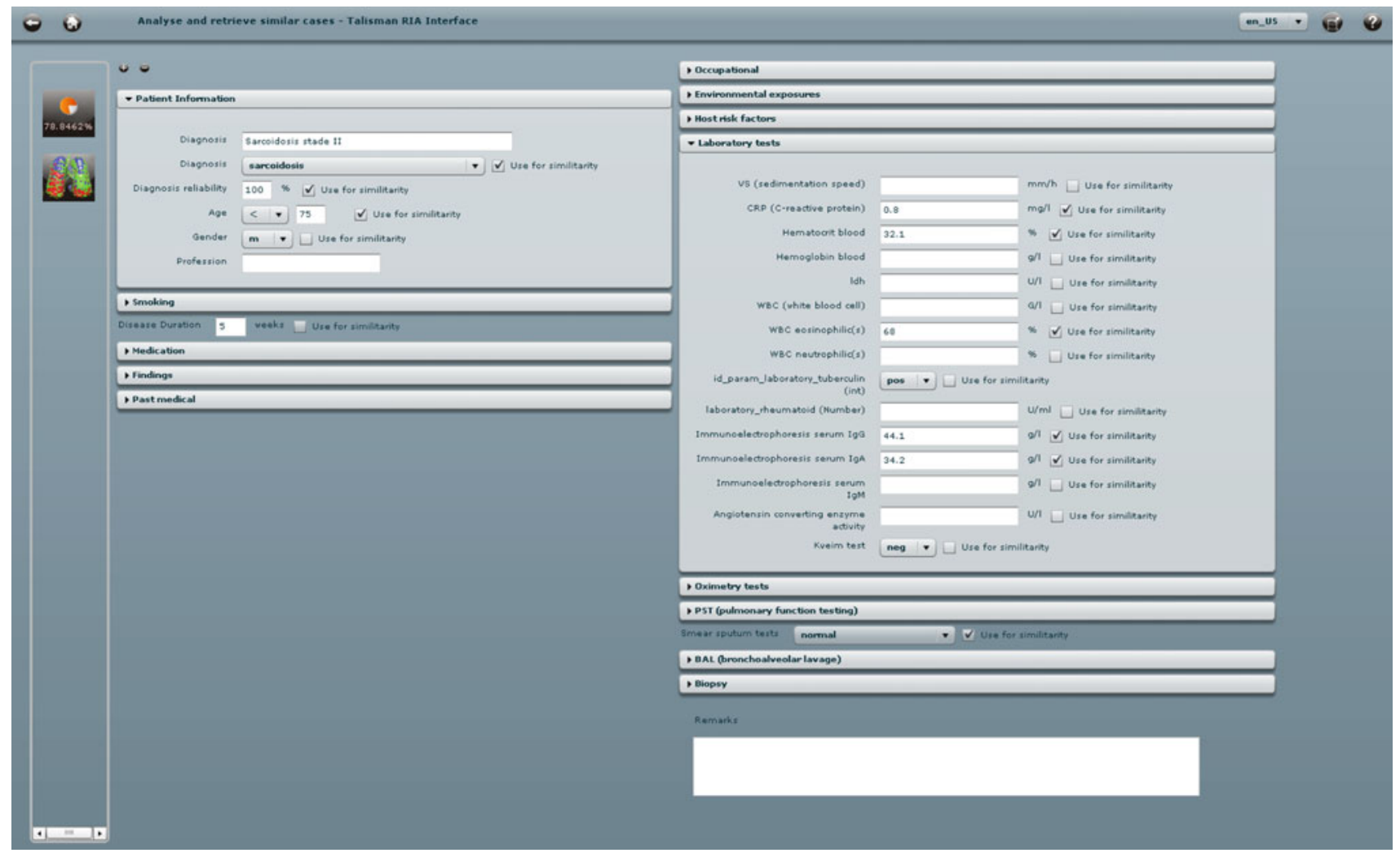

Fig. 12 Selection of the clinical parameters for case-based retrieval. A multimodal distance described in section "Similar case retrieval" is used (see Eq. 1). The visual modality is included only if a query image series was previously analyzed

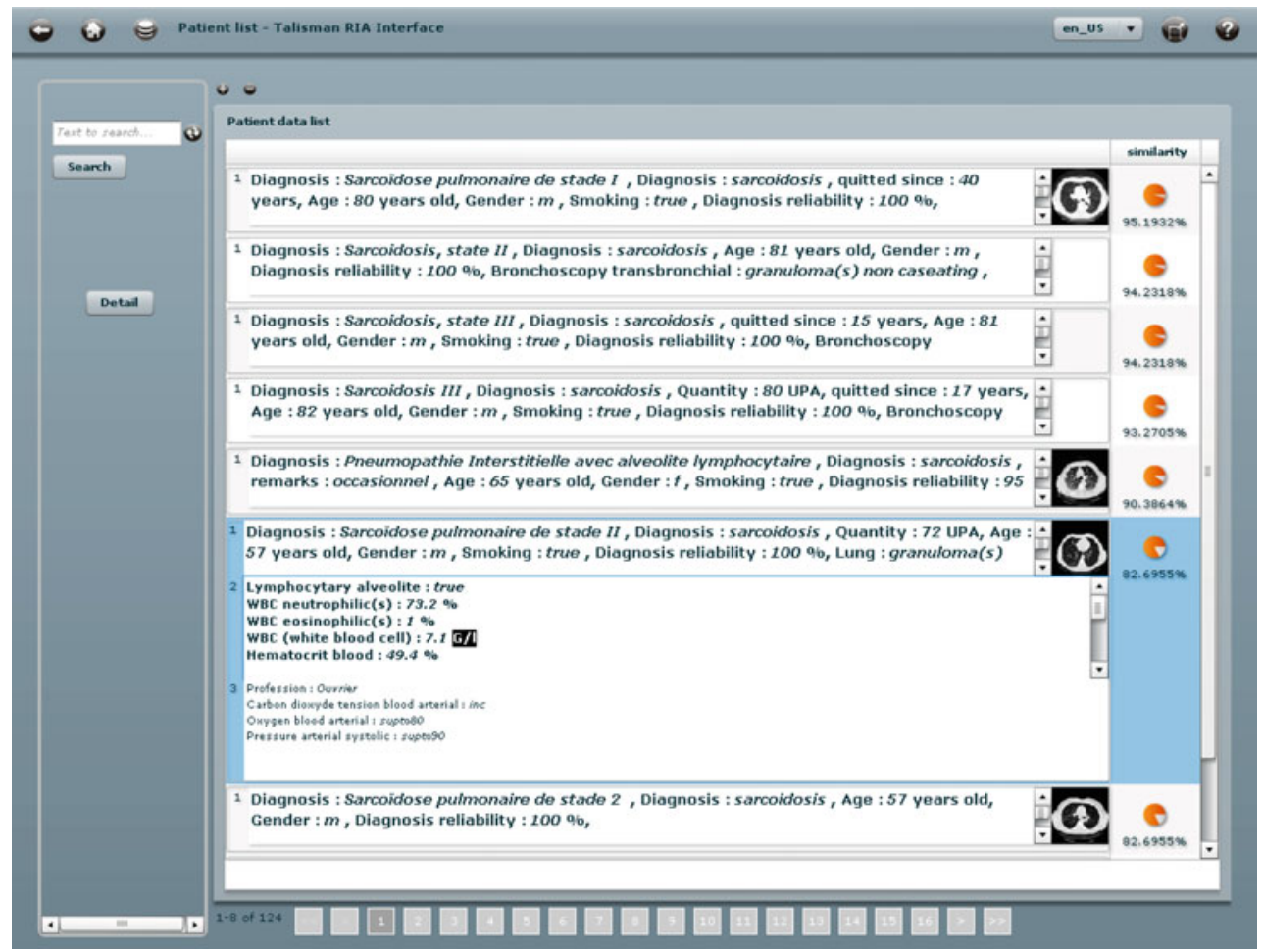

Fig. 13 List of cases returned by the multimodal query. The similarity measure is displayed using pie charts. Similarly to the "browse" use case, detailed views of a selected case are available by clicking the "Detail" button to access pages depicted in Figs. 6 and 7 
Table 1 Detailed performance in $\%$ of the blockwise classification of the various lung tissue types

\begin{tabular}{llll}
\hline & Sensitivity & Specificity & Accuracy \\
\hline Healthy & 63.04 & 92.16 & 87.94 \\
Emphysema & 74.67 & 98.46 & 96.3 \\
Ground glass & 62.53 & 94.75 & 86.51 \\
Fibrosis & 86.22 & 89.99 & 89.02 \\
Micronodules & 77.65 & 90.72 & 87.43 \\
\hline
\end{tabular}

It is important to note that the overlaid display only shows the most probable lung tissue pattern as second opinion. The user can access original data anytime by adjusting the transparency of the color map to assess local reliability of the proposed output. The computation time required to categorize a whole HRCT scan with 29 slices and a distance between the center of the blocks of 4 pixels is 19.4 min on a desktop PC with Intel 17@2.67 GHz CPU and 6GB of memory.

The third use case for similar case retrieval is useful both for radiologists and for physicians, as it deals not only with radiological findings but also with final histological diagnoses. The range of the diagnoses of the retrieved cases provides information to the clinician with detailed examples. Again, a LOPO CV based on cases as instances was used to compute the retrieval performance of the proposed system. An average precision of the first retrieved case (P@1) of 59.4\% was obtained when evaluated based on the seven most represented histological diagnoses available in the database [6].
For diagnoses where the spatial distribution is important, improvements in early precision were observed when a local comparison of the proportions of lung tissue types is used instead of the global approach (i.e., Eq. 2) [8]. It is also important to keep in mind that the link between visual similarity of two HRCT scans and their associated diagnoses is not straightforward, as shown in Fig. 14. A system showing cases that are visually similar but with distinct diagnoses prevents the reader from mixing diagnoses with similar radiological findings, which is difficult to assess with the quantitative evaluation. This use case enables objective inter-case similarity assessment. Similarity can be tailored according the user's need where the inter-case distance can be based on either filled clinical parameters or visual content or on both modalities. The proposed system is limited to HRCT images with 1 millimeter $(\mathrm{mm})$ collimation at $10 \mathrm{~mm}$ intervals and is not able to compare images with various CT technical parameters. Such a functionality could be imagined in the future but would require significant effort.

The success of the proposed CAD system also depends on its acceptance in a clinical environment. Particular care was taken to disturb classical clinical workflows as little as possible. The approach of the clinician to a diagnosis if he has little experience of the domain is to compare the image under investigation with typical cases with confirmed diagnosis listed in textbooks or contained in personal collections and the use case for similar case retrieval aims at easing and standardizing the visual information search. However, a risk of inducing false alarms to the radiologists is also present

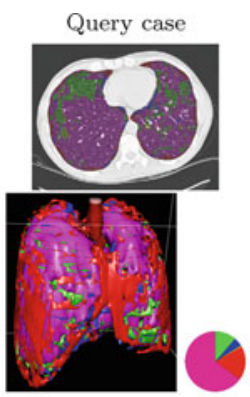

(a) Tuberculosis

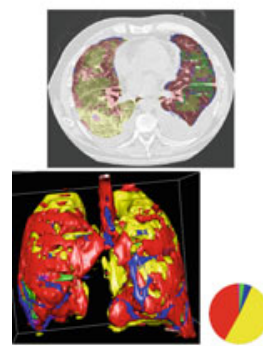

(a) Pneumocystis pneumonia

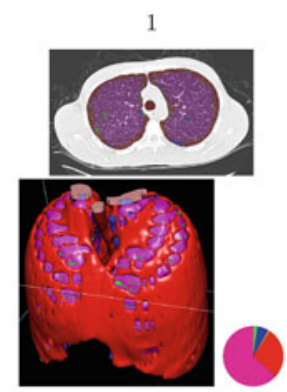

(b) Tuberculosis

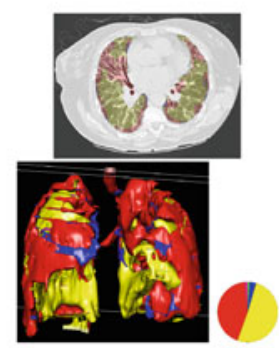

(b) Pulmonary fibrosis

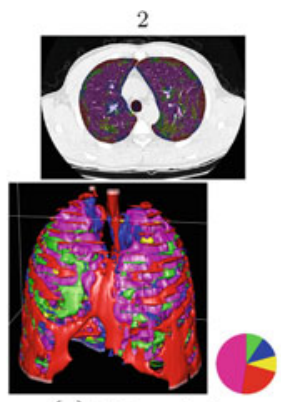

(c) Tuberculosis

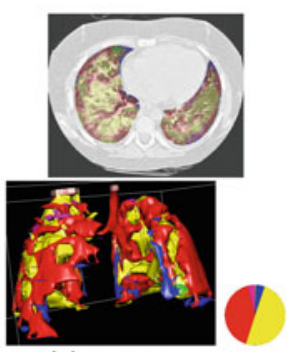

(c) Pneumocystis pneumonia

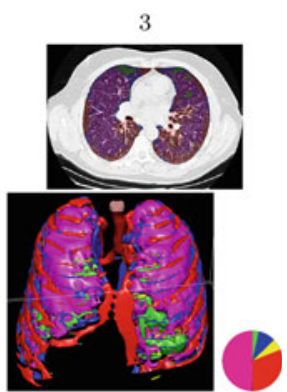

(d) Sarcoidosis

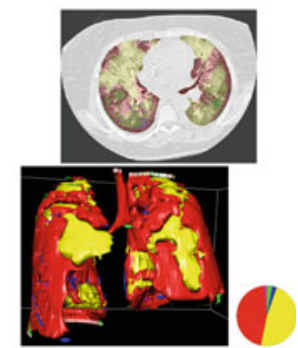

(d) Acute interstitial pneumonia

Fig. 14 Retrieval examples using $d_{\text {visual }}$. Pie charts show the distribution of the various lung tissue types (i.e., healthy in green, emphysema in blue, ground glass in yellow, fibrosis in red and micronodules in pink.) 
as only two healthy cases are contained in the database and the retrieval system is likely to inaccurately retrieve positive cases.

\section{Conclusions}

In this work, the clinical workflows and GUIs of a webbased hybrid detection-CBIR-based CAD system to assist the diagnosis of ILDs are proposed. Most of the systems found in the literature are proposing computer-aided detection of the abnormal lung tissue, letting the radiologists down with the problem of the final histological diagnosis. The implemented clinical workflows show that detection-based and CBIR-based CAD are complementary both on the user's side and on the algorithmic side. The next steps are the evaluation of the system in clinical routine to assess the performance of the radiologists with and without the system.

Acknowledgments This work was supported by the Swiss National Science Foundation (FNS) with grant 205321-130046 as well as the EU $7^{\text {th }}$ Framework Program in the context of the Khresmoi project (FP7-257528).

\section{Conflict of interest None.}

\section{References}

1. Abe H, MacMahon H, Engelmann R, Li Q, Shiraishi J, Katsuragawa S, Aoyama M, Ishida T, Ashizawa K, Metz CE, Doi K (2003) Computer-aided diagnosis in chest radiography: results of large-scale observer tests at the 1996-2001 RSNA scientific assemblies. RadioGraphics 23(1):255-265

2. Aisen A, Broderick L, Winer-Muram H, Brodley C, Kak A, Pavlopoulou C, Dy J, Shyu C, Marchiori A (2003) Automated storage and retrieval of thin-section CT images to assist diagnosis: system description and preliminary assessment. Radiology 228(1):265-270

3. Caritá EC, Seraphim E, Honda MO, Mazzoncini de AzevedoMarques P (2008) Implementation and evaluation of a medical image management system with content-based retrieval support. Radiol Brasileira 41(5):331-336

4. Depeursinge A, Müller H (2010) Fusion techniques for combining textual and visual information retrieval. In: ImageCLEF, The springer international series on information retrieval, vol. 32 . Springer, Berlin Heidelberg, pp 95-114

5. Depeursinge A, Racoceanu D, Iavindrasana J, Cohen G, Platon A, Poletti PA, Müller H (2010) Fusing visual and clinical information for lung tissue classification in high-resolution computed tomography. Artif Intell Med 50(1):13-21

6. Depeursinge A, Vargas A, Platon A, Geissbuhler A, Poletti PA, Müller H (2010) 3D case-based retrieval for interstitial lung diseases. In: MCBR-CDS 2009: Medical content-based retrieval for clinical decision support, lecture notes in computer science (LNCS). Springer, pp 39-48

7. Depeursinge A, Vargas A, Platon A, Geissbuhler A, Poletti PA, Müller $\mathrm{H}$ Building a reference multimedia database for interstitial lung diseases. Comput Med Imaging Graph (to appear)
8. Depeursinge A, Zrimec T, Busayarat S, Müller H (2011) 3D lung image retrieval using localized features In: Medical imaging 2011: computer-aided diagnosis, vol. 7963. SPIE, p 79632E

9. Doi K (2007) Computer-aided diagnosis in medical imaging: historical review, current status and future potential. Comput Med Imaging Graph 31(4-5):198-211

10. Duncan JS, Ayache N (2000) Medical image analysis: progress over two decades and the challenges ahead. IEEE Trans Pattern Anal Mach Intell 22(1):85-106

11. Engle RL (1992) Attempts to use computers as diagnostic aids in medical decision making: a thirty-year experience. Perspect Biol Med 35(2):207-219

12. Friedman C, Elstein A, Wolf F, Murphy G, Franz T, Heckerling P, Fine P, Miller T, Abraham V (1999) Enhancement of clinician's diagnostic reasoning by computer-based consultation. J Am Med Assoc 282(19):1851-1856

13. Gallo L, De Pietro G, Coronato A, Marra I (2008) Toward a natural interface to virtual medical imaging environments. In: AVI '08: Proceedings of the working conference on advanced visual interfaces, Association for Computing Machinery. New York, pp 429-432

14. Hoffman EA, Reinhardt JM, Sonka M, Simon BA, Guo J, Saba O, Chon D, Samrah S, Shikata H, Tschirren J, Palagyi K, Beck KC, McLennan G (2003) Characterization of the interstitial lung disease via density-based and texture-based analysis of computed tomography images of lung structure and function. Acad Radiol 10(10):1104-1118

15. Kelly K, Dean J, Lee SJ, Comulada W (2010) Breast cancer detection: radiologists' performance using mammography with and without automated whole-breast ultrasound. Eur Radiol 20:25572564

16. Keysers D, Dahmen J, Ney H, Wein BB, Lehmann TM (2003) A statistical framework for model-based image retrieval in medical applications. J Electron Imaging 12(1):59-68

17. King TE (2010) Approach to the adult with interstitial lung disease: clinical evaluation. In: Denise S. Basow (edn). UpToDate, Waltham, MA

18. Kruger RP, Thompson WB, Turner AF (1974) Computer diagnosis of pneumoconiosis. IEEE Transact Syst Man Cybern SMC$4(1): 40-49$

19. Lemaire JB, Schaefer JP, Martin LA, Faris P, Ainslie MD, Hull RD (1999) Effectiveness of the quick medical reference as a diagnostic tool. Can Med Assoc J 161(6):725-728

20. Liu CT, Tai PL, Chen AYJ, Peng CH, Wang JS (2000) A contentbased medical teaching file assistant for CT lung image retrieval. In: Proceedings of the IEEE international conference on electronics, circuits, systems. Jouneih-Kaslik, Lebanon, pp 361-365

21. Mazoue JG (1990) Diagnosis without doctors. J Med Philos 15(6):559-579

22. Meyers P, Nice C, Becker H, Nettleton W, Sweeney J, Meckstroth GR (1964) Automated computer analysis of radiographic images. Radiology 83:1029-1034

23. Miller RA (1994) Medical diagnostic decision support systemspast, present, and future: a threaded bibliography and brief commentary. J Am Med Inform Assoc 1(1):8-27

24. Müller H, Michoux N, Bandon D, Geissbuhler A (2004) A review of content-based image retrieval systems in medicine-clinical benefits and future directions. Int J Med Inform 73(1):1-23

25. Müller H, Rosset A, Garcia A, Vallée JP, Geissbuhler A (2005) Benefits from content-based visual data access in radiology. RadioGraphics 25(3):849-858

26. Nishikawa RM (2007) Current status and future directions of computer-aided diagnosis in mammography. Comput Med Imaging Graph 31(4-5):224-235 
27. Oliveira MC, Cirne W, de Azevedo Marques PM (2007) Towards applying content-based image retrieval in the clinical routine. Future Gener Comput Syst 23(3):466-474

28. Rosset A, Spadola L, Ratib O (2004) OsiriX: an open-source software for navigating in multidimensional DICOM images. J Digit Imaging 17(3):205-216

29. Salgado R, Mulkens T, Bellinck P, Termote JL (2003) Volume rendering in clinical practice. a pictorial review. J Belge de Radiologie 86(4):215-220

30. Sasso G, Marsiglia HR, Pigatto F, Basilicata A, Gargiulo M, Abate AF, Nappi M, Pulley J, Sasso FS (2005) A visual queryby-example image database from chest $\mathrm{CT}$ images: potential role as a decision and educational support tool for radiologists. J Digit Imaging 18(1):78-84

31. Schroeder W, Martin K, Lorensen B (2006) The visualization toolkit - an object oriented approach to 3D graphics. 3rd edn. Kitware, Inc, Clifton park

32. Shyu CR, Brodley CE, Kak AC, Kosaka A, Aisen AM, Broderick LS (1999) ASSERT: a physician-in-the-loop contentbased retrieval system for HRCT image databases. Comput Vis Imag Underst 75(1/2):111-132

33. Simel D, Drummond R (2008) The rational clinical examination: evidence-based clinical diagnosis. McGraw-Hill, NY

34. Sluimer IC, van Waes PF, Viergever MA, van Ginneken B (2003) Computer-aided diagnosis in high resolution CT of the lungs. Med Phys 30(12):3081-3090
35. Smeulders AWM, Worring M, Santini S, Gupta A, Jain $\mathrm{R}$ (2000) Content-based image retrieval at the end of the early years. IEEE Trans Pattern Anal Mach Intell 22(12):1349-1380

36. Tao Y, Zhou XS, Bi J, Jerebkoa A, Wolf M, Salganicoff M, Krishnana A (2009) An adaptive knowledge-driven medical image search engine for interactive diffuse parenchymal lung disease quantification. In: Medical imaging 2009: computer-aided diagnosis, vol 7260. SPIE, p 726007

37. Uchiyama Y, Katsuragawa S, Abe H, Shiraishi J, Li F, Li Q, Zhang CT, Suzuki K, Doi K (2003) Quantitative computerized analysis of diffuse lung disease in high-resolution computed tomography. Med Phys 30(9):2440-2454

38. Vomweg TW (2008) Computer-aided diagnosis: clinical applications in the breast. In: Image processing in radiology, medical radiology. Berlin Heidelberg, Springer, pp 355-374

39. Warner H, Bouhaddou O (1994) Innovation review: iliad-a medical diagnostic support program. Top Heal Inf Manag 14(4):51-58

40. Webb WR, Müller NL, Naidich DP (2001) High-Resolution CT of the lung. Lippincott Williams \& Wilkins, Philadelphia

41. Welter P, Deserno TM, Fischer B, Wein BB, Ott B, Günther RW (2009) Integration of CBIR in radiological routine in accordance with IHE. In: Medical imaging 2009: Advanced PACS-based imaging informatics and therapeutic applications, vol 7264. SPIE, p 726404 